\title{
Dynamische Geometrie ohne Computer: Die mathematischen Trickfilme des Geheimen Schulrats Münch
}

\author{
Sebastian Kitz
}

Eingegangen: 25. Februar 2013 / Angenommen: 17. März 2013 / Online publiziert: 22. Juni 2013

(C) Springer-Verlag Berlin Heidelberg 2013

\section{Der Einzug der Beweglichkeit in den Geometrieunterricht}

Im letzten Drittel des 19. Jahrhunderts wurde vermehrt kritisiert, dass sich der damals praktizierte Geometrieunterricht weitgehend an den Elementen Euklids orientierte. Die Kritik konzentrierte sich dabei im Wesentlichen auf zwei Hauptpunkte: einerseits die fehlende Verbindung von Planimetrie und Stereometrie, andererseits die Starrheit und Unbeweglichkeit der geometrischen Figuren und damit einhergehend des ganzen Systems der Geometrie. Nach Meinung der Kritiker war letzteres der Hauptgrund für den unnatürlichen Aufbau der Euklidischen Geometrie, der sich allein aus logischen Gesichtspunkten ergebe. Außerdem vertraten sie die These, dass sich die Geometrie durch die Verwendung beweglicher Figuren wesentlich vereinfachen ließe und eine große Zahl von Sonderfällen beseitigt werden könnte. ${ }^{1}$

Um die genannten Schwächen zu beheben, kam der Vorschlag auf, einige Elemente der damals so genannten Neueren Geometrie ${ }^{2}$ in den Schulunterricht aufzunehmen. Dieser Vorschlag wurde sehr kontrovers diskutiert, z.B. in den ersten Jahrgän-

\footnotetext{
${ }^{1}$ Man vergleiche dazu [14] und [18].

${ }^{2}$ Darunter wurde im Wesentlichen projektive Geometrie im Sinne Jacob Steiners verstanden. Manche Reformer orientierten sich auch an von Staudt. Neben der Bezeichnung Neuere Geometrie (als Abgrenzung zur alten Euklidischen Geometrie) waren auch die Begriffe Geometrie der Lage (im Gegensatz zur Geometrie des Maßes) und Synthetische Geometrie (im Gegensatz zur Analytischen Geometrie) üblich.

S. Kitz $(\bowtie)$

AG Didaktik und Geschichte der Mathematik, Bergische Universität Wuppertal, 42097 Wuppertal, Deutschland

e-mail: kitz@uni-wuppertal.de
} 
gen der „Zeitschrift für mathematischen und naturwissenschaftlichen Unterricht“ ${ }^{\text {3 }}$ oder auf der „Versammlung deutscher Philologen und Schulmänner“ 1876 und in den Folgejahren. ${ }^{4}$

Letzten Endes waren die Bemühungen der Reformer dennoch von Erfolg gekrönt, da die diskutierten Inhalte als Unterrichtsgegenstand für die oberen Klassen des Gymnasiums in die Lehrpläne einiger deutscher Länder aufgenommen wurden. ${ }^{5}$ Der Einfluss beschränkte sich allerdings nicht auf die genannten Oberklassen, sondern wirkte sich auch auf die Methode beim Unterricht der bestehenden Inhalte aus. So unterschied beispielsweise der Mathematikdidaktiker Lietzmann ${ }^{6} 1909$ in seiner Abhandlung „Stoff und Methode im mathematischen Unterricht der norddeutschen höheren Schulen“ explizit zwischen „Beweglichkeit der Figuren. Geometrie der Lage auf der Unterstufe“ und „Geometrie der Lage auf der Oberstufe“, wobei er unter letzterem die eigentlich von den Reformern angestrebte Einführung neuer Inhalte verstand. Kennzeichnend für die „Geometrie der Lage auf der Unterstufe“ war für ihn:

„Da sind zunächst die Begriffsbestimmungen zu nennen. Linien werden als Orte bewegter Punkte erklärt (z.B. der Kreis), der Winkel entsteht durch Drehung eines Strahles um seinen Endpunkt; auch die Unterscheidung Gerade-StrahlStrecke und damit Dreiseit-Dreieck, Vierseit-Viereck ist nicht euklidisch. Damit steht im Zusammenhang die Einführung positiver und negativer Strecken, wie sie zwar in der Trigonometrie allgemein gebräuchlich ist, während wir ihr in der Planimetrie nur selten begegnen. (...) Die axiale Symmetrie findet in immer mehr Lehrbüchern Eingang. (...) Die Benutzung zentraler Symmetrie ist nicht ganz so häufig. (...) Die Veränderlichkeit der einzelnen Stücke in einer Figur, insbesondere, wie wir schon früher sahen, bei den Konstruktionsaufgaben, läßt die gegenseitige Abhängigkeit erkennen und ist deshalb außerordent-

\footnotetext{
${ }^{3}$ Man vergleiche z.B. [4], [8] und [16].

${ }^{4}$ Man vergleiche [6] und [7].

${ }^{5} \mathrm{Vgl}$. dazu [2], [10], [17] und [20].

${ }^{6}$ Walther Lietzmann (1880-1959) arbeitete nach seiner Promotion bei Hilbert ab 1906 als Lehrer an der Oberrealschule Barmen. 1914 wurde er Direktor der Oberrealschule in Jena, 1919 ging er in gleicher Position nach Göttingen. Er wirkte wesentlich an Felix Kleins Reformprogramm für den Mathematikunterricht mit und brachte eine Vielzahl von Lehrwerken heraus.
}
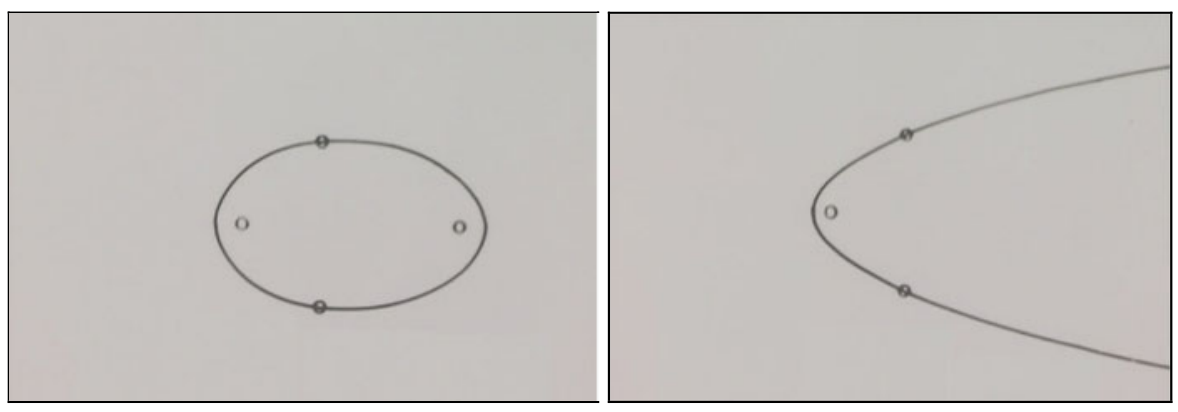
lich geeignet, die Bildung des Funktionsbegriffs zu unterstützen." (Lietzmann [11, S. 35-36])

In der Folgezeit entwickelte der Ansatz, die alten Inhalte nach der neuen Methode zu unterrichten, ein starkes Eigenleben. Dazu trugen nicht zuletzt die Meraner Reformvorschläge und die darin vorgesehene Stärkung des funktionalen Denkens bei, das die Reformer auch in der Geometrie durch die beweglichen Figuren fördern wollten. So war wohl letztlich die Verwendung der neuen Methode weitgehender akzeptiert als die Berücksichtigung der neuen Inhalte. Dies lässt sich unter anderem daran sehen, dass in den 1920er Jahren auf Grund einer radikalen Kürzung der Unterrichtszeit für Mathematik und Naturwissenschaften und damit einhergehend der zu vermittelnden Inhalte die Elemente der Neueren Geometrie aus den Lehrplänen verschwanden, der Aspekt der Beweglichkeit innerhalb der Geometrie jedoch ausdrücklich erhalten blieb. ${ }^{7}$

Neben der Möglichkeit, die Figuren rein in der Vorstellung beweglich zu machen, wurden im Lauf der Zeit verschiedene Methoden bzw. Lehrmittel zur Veranschaulichung entwickelt. Beispielsweise wurde durch Zeichnung einiger Situationen die Veränderung dargestellt (Abb. 1).

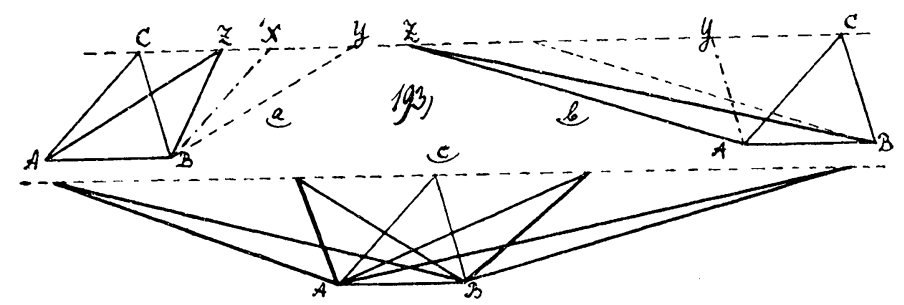

Abb. 1 „Verwandlung des Dreiecks und sein Inhalt“ (aus [18], Figurentafel)

Eine andere Option war der Einsatz von mechanischen Modellen. Diese wurden beispielsweise von Ernst Wienecke ${ }^{8}$ entwickelt, aus Holz und Metall gefertigt und über den Verlag Winckelmann in Berlin vertrieben (Abb. 2).

\footnotetext{
${ }^{7}$ Man vergleiche hierzu [9].

${ }^{8}$ Wienecke war Lehrer der Mathematik an der 5. Städtischen Fortbildungsschule in Berlin.
}
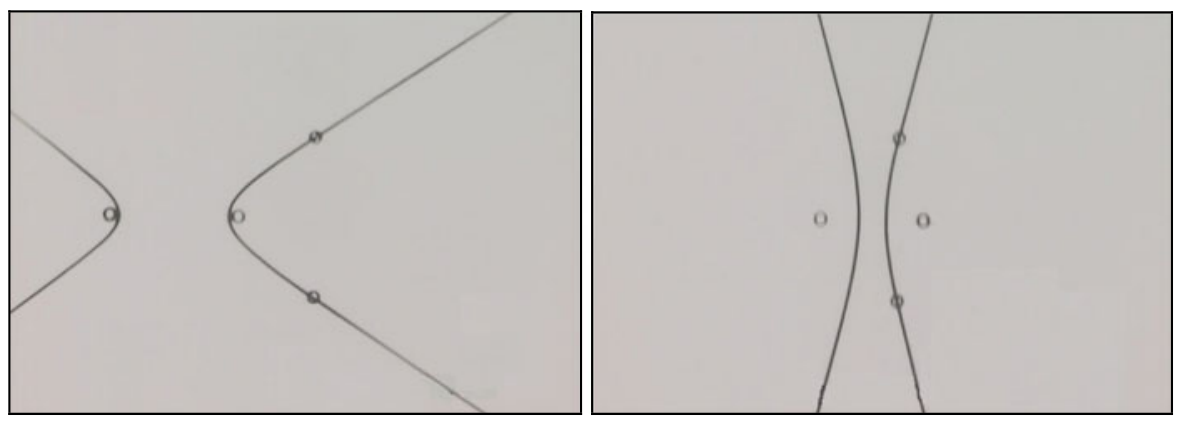


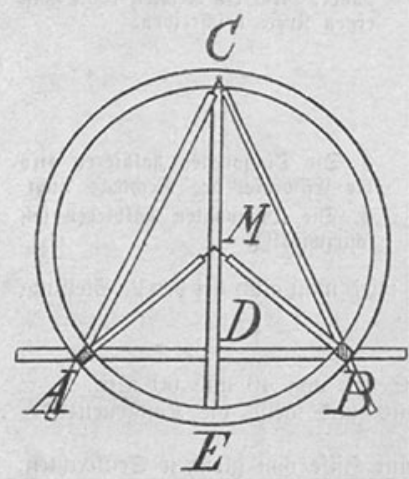

Abb. 2 Wieneckes Modell für Winkelsätze am Kreis (aus [19], 14)

Eine ähnliche Idee stand hinter der „Mathematischen Experimentiermappe“ von Gustav Noodt, ${ }^{9}$ die Bauteile aus Pappe enthielt, so dass die Schüler die Modelle selbst herstellen konnten (Abb. 3).

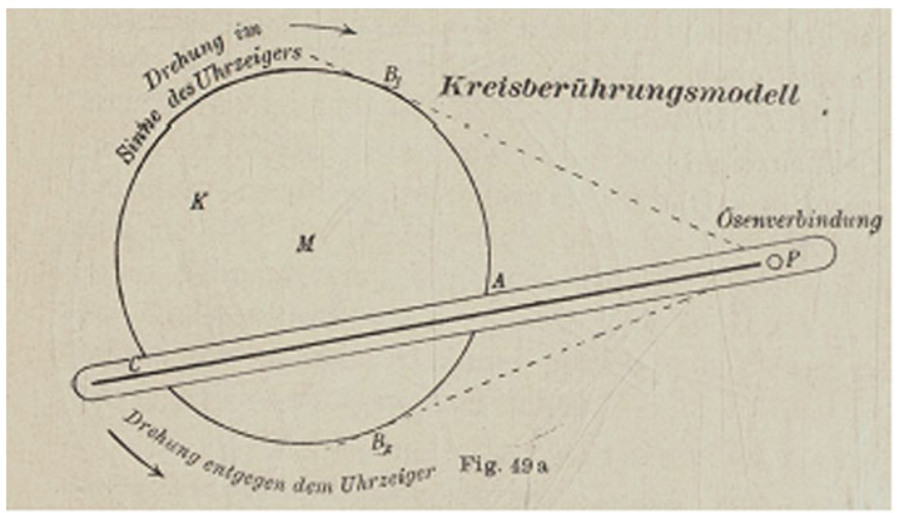

Abb. 3 Kreisberührungsmodell (aus [12], 20)

\begin{abstract}
${ }^{9}$ Noodt (1856-1917) studierte Mathematik und Naturwissenschaften in Göttingen und Berlin. Nach bestandenem Staatsexamen arbeitete er ab 1885 an Realgymnasien in Altona und Berlin. 1892 promovierte er an der Universität Rostock.
\end{abstract}
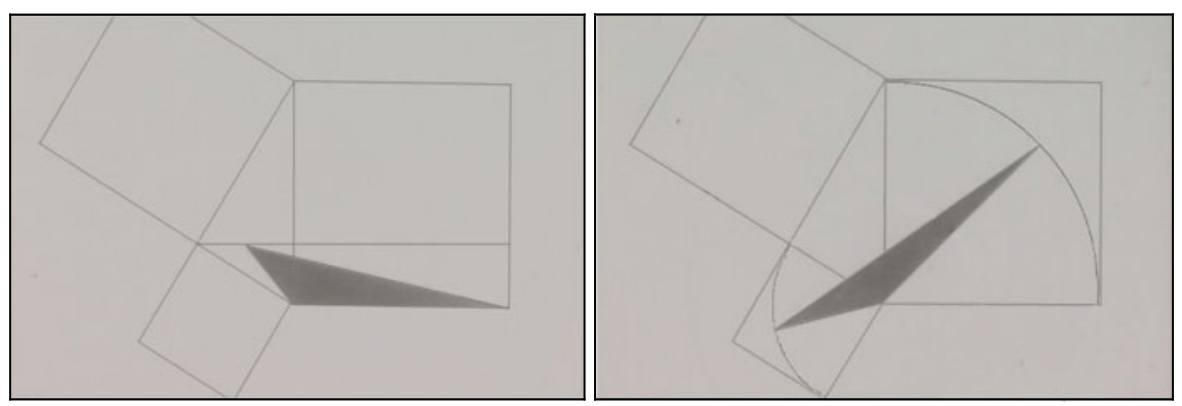
Als weiteres Hilfsmittel wurden Daumenkinos eingesetzt. Dabei handelt es sich um Bücher, die es ermöglichen, eine Serie von Einzelbildern schnell durchzublättern, so dass eine fortlaufende Bildfolge entsteht. Sind die Veränderungen von einem Bild zum nächsten richtig aufeinander abgestimmt, entsteht für den Betrachter der Eindruck einer kontinuierlichen Bewegung. Für den Mathematikunterricht wurden Daumenkinos beispielsweise von Hermann Detlefs ${ }^{10}$ unter der Bezeichnung „Geometrische Kinohefte“ entwickelt (Abb. 4).

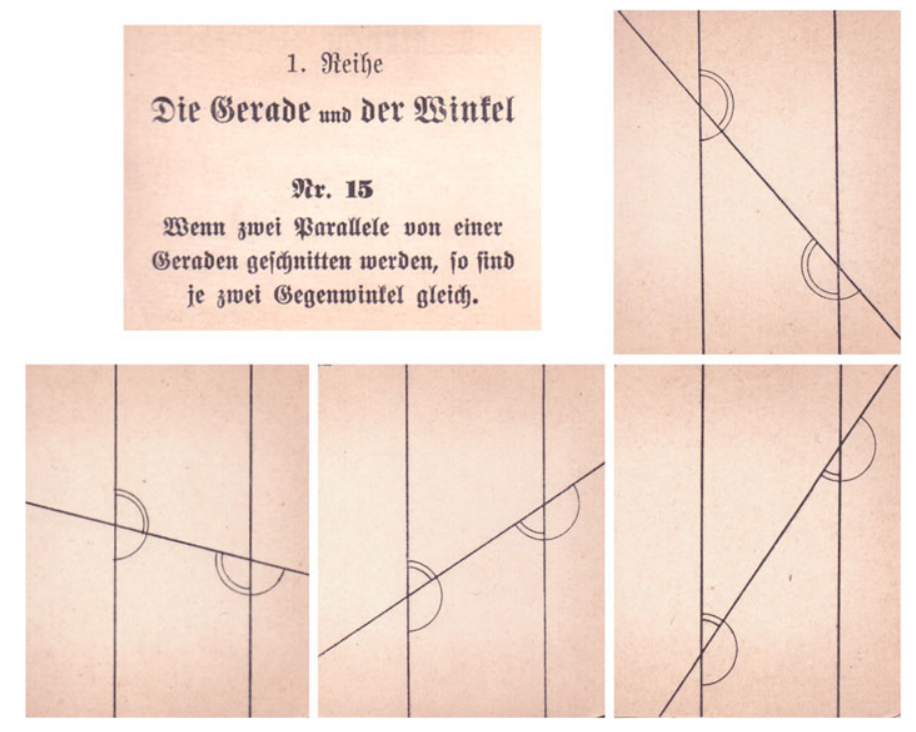

Abb. 4 Bildfolge (aus [3])

${ }^{10}$ Detlefs (1864-1927) studierte Mathematik, Physik und Geographie in Kiel und Berlin. Sein Examen legte er 1888 ab und arbeitete danach an Gymnasien und Realgymnasien u.a. in Kassel, Hamburg und Gevelsberg.
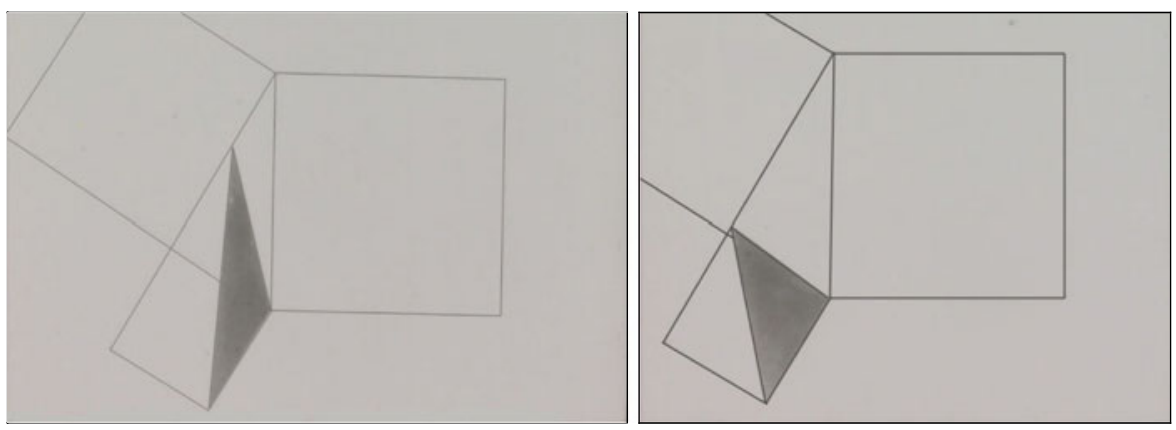


\section{Ludwig Münch und seine Filme}

Zu den genannten Lehrmitteln gehörten auch die mathematischen Trickfilme, die der Geheime Schulrat Ludwig Münch (1852-1922) aus Darmstadt ab 1910 herstellte. $^{11}$

Nach dem Studium der Philologie, Mathematik und Physik wurde Münch 1877 Lehrer am Realgymnasium in Darmstadt. Er wurde 1886 Direktor der Realschule zu Wimpfen am Berg, kehrte aber 1892 als Professor an das neue Gymnasium nach Darmstadt zurück, wo er zwei Jahre später Direktor an seiner alten Wirkungsstätte, dem Realgymnasium, wurde. Die Idee zur Herstellung von mathematischen Trickfilmen kam Münch, als er kurz nach der Jahrhundertwende in Stuttgart eine Filmvorführung erlebte.

Daraufhin begann er mit der Arbeit an seinem ersten Film, der den Euklidischen Beweis des Satzes von Pythagoras darstellte und erst 1910 vollendet wurde. Hilfe erhielt Münch dabei von seinem Kollegen Dr. Friedrich Pfersdorff und seinem Schwiegersohn Otto Hauck, die gemäß seiner Vorgaben die Hauptphasen zeichneten. Zum Zeichnen der Zwischenphasen ${ }^{12}$ engagierte Münch einen professionellen Zeichner (vermutlich ein Mitarbeiter der Firma Cartharius-Film, Darmstadt). Zudem nutzte er seine Position als Direktor des Realgymnasiums, indem er seine Studienreferendare zum Zeichnen abordnete. Auch ehemalige Schüler boten ihre Hilfe an. ${ }^{13}$

So entstand im Lauf der Zeit Material für 30 Filme, die dann von der Projektionsgesellschaft Union in Frankfurt am Main aufgenommen und vertrieben wurden. Der Preis pro Meter Film lag bei zwei Mark, wobei jeder Film eine Länge von 15 bis 20 Metern hatte. Ein entsprechender Projektionsapparat kostete 300 Mark. ${ }^{14}$ Die behandelten Themen waren neben dem bereits genannten Beweis des Pythagoras:

\footnotetext{
${ }^{11}$ Informationen zu Münch finden sich in zwei Meldebögen der Polizeilichen Melderegistratur der Stadt Darmstadt (Stadtarchiv Darmstadt, ST 12/18) sowie in den Beilagen des Großherzoglich-Hessischen Regierungsblatts von 1877 bis 1922. S.a. Pfersdorff und Hauck (in [5]).

${ }^{12}$ Pro Film waren 200 bis 400 Bilder erforderlich.

${ }^{13}$ Den Vorwurf, er habe auch seine Schüler zum Zeichnen herangezogen, wies Münch im 54. Band des Pädagogischen Archivs entschieden zurück. Er betonte, „daß die sämtlichen Zeichnungen zu dem vorgeführten Film nicht von den unter seiner Leitung stehenden, sondern von ehemaligen Schülern seiner Anstalt angefertigt worden sind“ ([23], 107].

${ }^{14}$ Im Jahr 1910 lag in Nürnberg der Preis für ein Pfund Kartoffeln bei 4 Pfennigen, ein Pfund Kornbrot kostete 15 Pfennige und für einen Liter Milch musste man 20 Pfennige bezahlen (vgl. [22], 88).
}
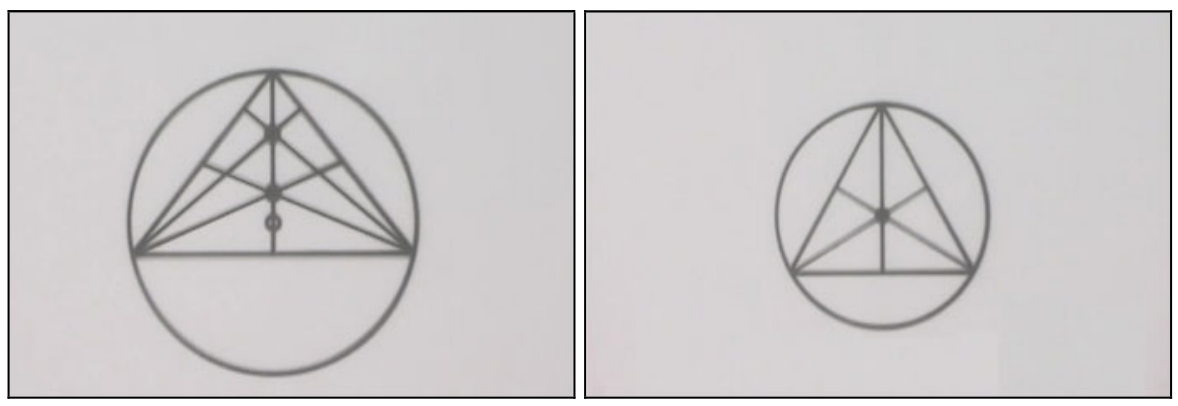
- das Apollonische Berührproblem

- der Übergang verschiedener Kegelschnitte ineinander („Metamorphose“)

- Kegelschnittsysteme, welche vier Bedingungen genügen (das sind z.B. alle Kegelschnitte, welche durch vier feste Punkte verlaufen)

- Abrollen des Krümmungskreises entlang einer Kurve, Spur des Mittelpunktes (Evolute)

- Darstellung der Planetenbewegung nach Ptolemäus und Kopernikus.

Schon bald nach Fertigstellung der ersten Filme begann Münch damit, diese öffentlich vorzuführen. Er tat dies sowohl auf lokaler Ebene als auch bei größeren Tagungen. Der Sinn dieser Vorführungen war einerseits, für den Einsatz der Filme im Mathematikunterricht zu werben. Andererseits nutzte Münch die dabei eingenommenen Honorare zur Finanzierung von weiteren Filmen. Die Reaktion des Publikums und der Öffentlichkeit war weitestgehend positiv. Geradezu überschwänglich berichtete die Zeitschrift „Licht-Bild-Bühne“ im Jahr 1912 mehrfach über Vorträge Münchs, so auch über den Vortrag beim Naturwissenschaftlichen Verein Darmstadt vom 13.2.1912:

„Im Kinematographen entwickelte sich alles wie im Wachstum der Natur, es kehrte vom Gipfelpunkte der Entwicklung zurück zu der ersten Form, und man gewann, nicht ohne eine gewisse innere Erschütterung, die feste Ueberzeugung, daß hier im kleinen, wie in der Gesamtnatur, sich die ewigen ehernen Gesetze vor den Augen des Beschauers vollziehen. (...) Die in allen Stücken glänzend verlaufene Vorführung ist als ein epochemachendes Ereignis für die Popularisierung der Wissenschaft zu betrachten, welches die aufmerksamste Beachtung der mathematischen Welt beanspruchen muß“ ([21], 10-12).

Und die „Frankfurter Zeitung“ schrieb über eine Vorführung Münchs am UnionTheater in Frankfurt a. M. vom 7.4.1912:

„In dem bronzierten Bühnenrahmen des Uniontheaters auf der Zeil erschienen am Sonntag Vormittag ,lebende Bilder“ eigener Art. Was sonst vielleicht im Traum gequälten Schülerhirnen geschehen mag, das ward hier zur Wirklichkeit. Der pythagoräische Lehrsatz begann lebendig zu werden. Der Beweis des seligen Euklides marschierte munter vor aller Augen daher und leuchtete auch fanatischen Nichtmathematikern ein. Kreise, Tangenten, Hyperbeln, Parabeln folgten in allen möglichen verwickelten Beziehungen, und zum Schluß kamen
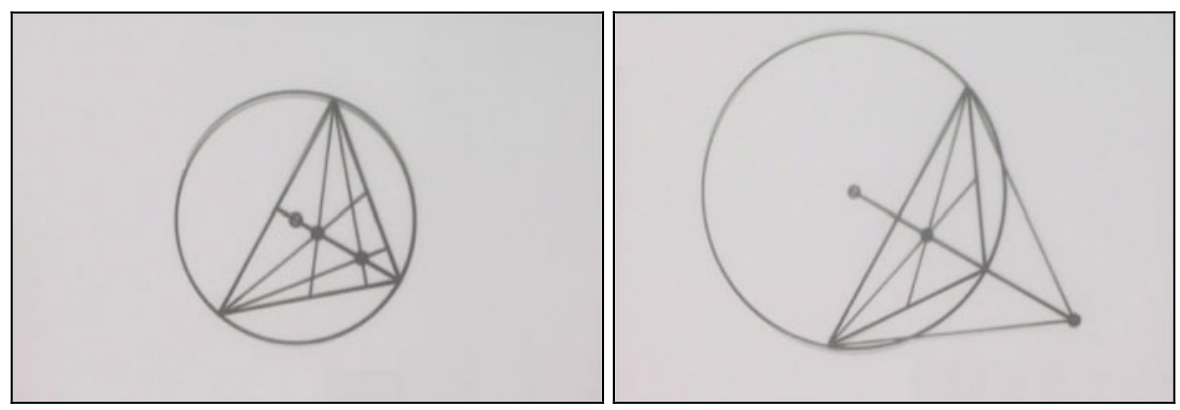
gar Dinge von abenteuerlicher Schwierigkeit, Aufgaben aus einem Gebiet der Mathematik, das man fast als transcendental bezeichnen kann. Und das alles, was noch keines Menschen Auge sah, was sich nicht einmal Universitätsprofessoren der Mathematik stets klar vorstellen können, erblickte man in verblüffender Wirklichkeit auf der weißen Leinwand. (...) (Münch) hat damit ein Anschauungsmaterial geschaffen, dessen Wert als unvergleichlich bezeichnet werden kann, namentlich für das Gebiet der höheren und höchsten Mathematik, dieses Schlüssels zu allen Wundern der Welt, zu allen Erfolgen der Technik, der Erd- und Himmelskunde.“

Beim Fachpublikum stießen die Trickfilme nicht immer auf solch unbegrenzt positive Reaktionen. So findet sich im 53. Band des Pädagogischen Archivs ein Bericht von Julius Ruska ${ }^{15}$ über Münchs Vortrag auf der 83. Versammlung deutscher Naturforscher und Ärzte, die 1911 in Karlsruhe stattfand:

„Geh. Schulrat Münch leitete seinen Vortrag damit ein, dass der Kinematograph an sich weder gut noch schlecht sei, und daß die Schulmänner davon eine gute Anwendung machen sollten, damit die schlechte in den Hintergrund gedrängt würde. Ich kann die Zuversicht nicht teilen, daß dem Kinematographentheaterunfug, der sich nachgerade zu einer öffentlichen Kalamität auswächst und schlimmer wirkt wie die Schundliteratur, durch ernste wissenschaftliche Darbietungen der Boden abgegraben werden kann. Am allerwenigsten wird es durch Darbietungen mathematischer Art geschehen; (...) Gegen ausgedehntere kinematographische Vorführungen in der Schule scheint mir insbesondere die auch von ärztlicher Seite hervorgerufene Gefahr für die Augen der Schüler zu sprechen. (...) Abgesehen von diesem Einwand und einigen pädagogischen Bedenken, die ich nicht berühren möchte, da ihre Erörterung an dieser Stelle zu weit führen würde, waren die Vorführungen selbst, darüber war nur eine Stimme, in hohem Grade interessant und dankenswert. (... ) Von verschiedenen Seiten wurde denn auch in der Diskussion auf die Bedeutung der kinematographischen Vorführungen für die Pflege des funktionalen Denkens und die

${ }^{15}$ Ruska (1867-1949) war von 1889 bis 1910 Lehrer für Mathematik und Naturwissenschaften am Kurfürst-Friedrich-Gymnasium in Heidelberg und von 1908 bis 1913 Herausgeber des „Pädagogischen Archivs“. In dieser Funktion berichtete er vielfach über die Jahrestagungen der mathematischnaturwissenschaftlichen Verbände.
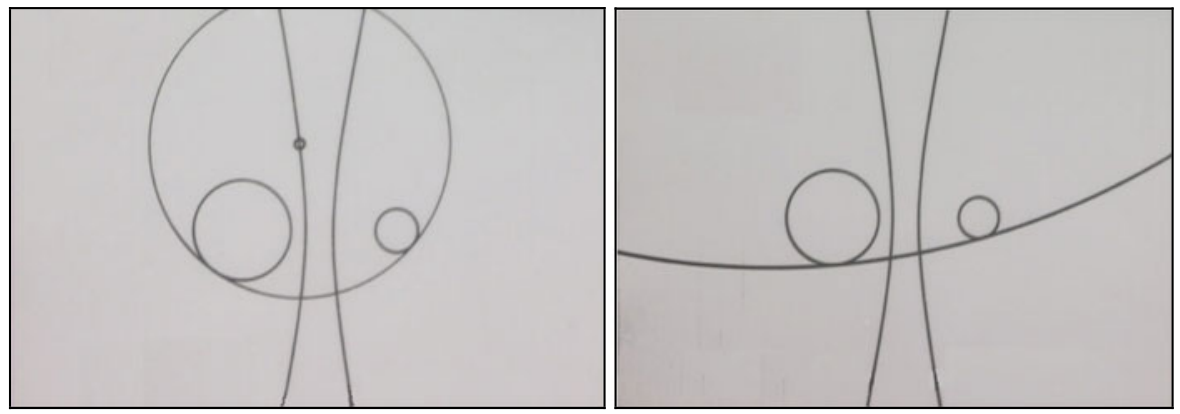
Stärkung des räumlichen Anschauungsvermögens, dann auch auf das ästhetisch Wertvolle der Darbietungen hingewiesen“ ([15], 663-664).

Positiver liest sich hingegen der Bericht von Alfred Postelmann ${ }^{16}$ über den „10. Ferienkursus für Lehrer höherer Schulen“, der vom 7. bis 19. Oktober 1912 in Frankfurt am Main stattfand. Dort wird über Münchs Vortrag gesagt:

„Von besonderem und eigenartigem Interesse war ein Vortrag von Geh. Schulrat Münch - Darmstadt: Das Kinematogramm im Dienste des Unterrichts in der niederen und höheren Mathematik. In geradezu dramatischer Anschaulichkeit führten die prächtigen Films - die auf Zehntausenden von einzelnen Zeichnungen beruhen! - das funktionale Leben geometrischer Figuren vor Augen, von der schlichten Kreistangente bis zu den verwickeltsten Kurvenscharen und kinomatischen Kurbelgetrieben, und auch der anfängliche Skeptiker erkannte bald, welch ein reicher Quell zur Belebung und Befruchtung unseres Unterrichts hier erschlossen werden wird, wenn - was Geh.-Rat Münch in baldige Aussicht stellen konnte - ein solches Hilfsmittel erst einmal Gemeingut aller werden kann“" ([13], 109).

Wie man aus den Reaktionen sieht, wurden die Filme damals von der großen Mehrheit des Publikums als durchaus sinnvolles Hilfsmittel für den Mathematikunterricht angesehen. Einer weiten Verbreitung stand wohl vor allem die fehlende technische Ausstattung in den Schulen entgegen. Es fehlte an Projektoren und abdunkelbaren Räumen. Zudem kam es damals oft zu Unfällen, weil bei Filmvorführungen Feuer ausbrach, denn das damals verwendete Trägermaterial Zelluloid ist leicht entflammbar, so dass schon bei kurzzeitigem Stillstand des Films vor der Projektionslampe auf Grund eines Defekts ein Brand entstehen konnte. Die Möglichkeit, für die Vorführung in bestehende Kino-Theater auszuweichen, wurde verworfen, da damit wohl eine zu große Nähe zu den Inhalten der dort normalerweise gezeigten Filme entstan-

${ }^{16}$ Postelmann (1880-1945) studierte an der Universität Königsberg Mathematik, Physik, Astronomie und Erdkunde. Nach bestandener Prüfung für das Lehramt an höheren Schulen arbeitete er an Gymnasien in Königsberg, Rastenburg und Bartenstein.
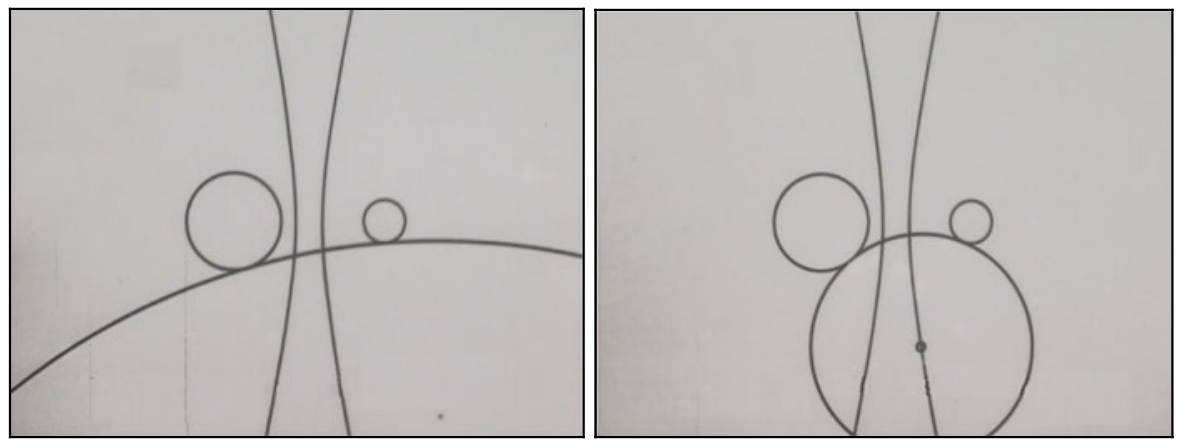
den wäre. ${ }^{17}$ Auch der Beginn des ersten Weltkrieges dürfte für die Beschaffung der notwendigen Ausstattung in den Schulen nicht förderlich gewesen sein.

Die hier gezeigten Bildfolgen können nur einen schwachen Eindruck von der Pionierarbeit geben, die Ludwig Münch vor 100 Jahren geleistet hat. Nach seinem Tod gerieten die Filme mehr und mehr in Vergessenheit. Heute existieren noch (teilweise unvollständige) Kopien von etwa 20 der ehemals 30 produzierten Filme im Bundesarchiv in Berlin und im Deutschen Filminstitut in Wiesbaden. Die Rechte liegen bei der Friedrich-Wilhelm-Murnau-Stiftung, Wiesbaden.

Münch war einerseits ein Vorreiter bei der Produktion von Trickfilmen, ${ }^{18}$ andererseits aber auch ein Vorbote der heute allgemein bekannten ,Dynamischen-GeometrieSoftware“. Bei aller Anerkennung für die Fortschritte, die in den letzten Jahren bei der Entwicklung solcher Systeme und ihrem Einsatz in der Schule gemacht wurden, sollten die mindestens genauso beachtlichen Leistungen des Geheimen Schulrats Ludwig Münch nicht vergessen werden.

\section{Literatur}

1. Beckerman, H.: Animation: The Whole Story. Allworth Press, New York (2003)

2. Cramer, H.: Der mathematische Unterricht an den höheren Schulen im Grossherzogtum Baden. Leipzig/Berlin (1910)

3. Detlefs, H.: Geometrische Kino-Hefte zur Veranschaulichung veränderlicher Figuren, 1. Reihe. Berlin (1913)

4. Fresenius, F.C.: Noch einmal die neuere Geometrie und die unendlich entfernten Gebilde. Z. Math. Naturwiss. Unterr. 2, 494-504 (1871)

5. Goebel, G.: Ein aufsehenerregender Fund. Der Film-Kreis. Heft 4(3), 24-25 (1958)

6. Günther, S.: Bericht über die Thätigkeit der mathematisch-naturwissenschaftlichen Sektion der 32. Versammlung deutscher Philologen und Schulmänner zu Wiesbaden. Z. Math. Naturwiss. Unterr. 9, 163-167 (1878)

7. Hauck, G.: Sitzungsbericht der mathematisch-naturwissenschaftlichen Section der 31. Versammlung deutscher Philologen und Schulmänner in Tübingen vom 25. bis 28 September 1876. Z. Math. Naturwiss. Unterr. 7, 510-520 (1876)

8. Kober, J.: Ueber das unendliche und die neuere Geometrie. Z. Math. Naturwiss. Unterr. 3, 249-264 (1872)

9. Lietzmann, W.: Die neuen mathematischen Lehrpläne für die höheren Knabenschulen in Preußen. Z. Math. Naturwiss. Unterr. 56, 193-207 (1925)

10. Lietzmann, W.: Die Organisation des mathematischen Unterrichts an den höheren Knabenschulen in Preussen. Leipzig/Berlin (1910)

11. Lietzmann, W.: Stoff und Methode im mathematischen Unterricht der norddeutschen höheren Schulen. Leipzig/Berlin (1909)

12. Noodt, G.: Leitfaden zur Mathematischen Experimentiermappe. Leipzig/Berlin (1912)

13. Postelmann, A.: Bericht über den 10. Ferienkursus für Lehrer höherer Schulen in Frankfurt a.M. Z. Math. Naturwiss. Unterr. 44, 108-110 (1913)

14. Reye, T.: Rede über die synthetische Geometrie im Alterthum und in der Neuzeit. Der Rectoratswechsel an der Kaiser-Wilhelms-Universität Strassburg am 1. Mai 1886. Strassburg (1886), S. $31-48$

15. Ruska, J.: Unterrichtsfragen auf der 83. Versammlung Deutscher Naturforscher und Ärzte in Karlsruhe. Pädagog. Archiv 53, 649-686 (1911)

\footnotetext{
${ }^{17}$ Über alle genannten Punkte berichtete die „Licht-Bild-Bühne“ mehrmals im 5. Jahrgang, z.B. in Nr. 3 , S. 13; Nr. 4, S. 23; Nr. 12, S. 16.

${ }^{18}$ Der erste gezeichnete Animationsfilm entstand 1906 in den USA, der vermutlich erste in Deutschland hergestellte Trickfilm wurde gegen Ende des Jahres 1909 uraufgeführt. Vgl. [1] und [24].
} 
16. Sturm, R.: Die neuere Geometrie auf der Schule. Z. Math. Naturwiss. Unterr. 1, 474-489 (1870)

17. Thaer, A., Geuther, N., Böttger, A.: Der mathematische Unterricht in den Gymnasien und Realanstalten der Hansestädte, Mecklenburgs und Oldenburgs. Leipzig/Berlin (1911)

18. Treutlein, P.: Der geometrische Anschauungsunterricht. Leipzig/Berlin (1911)

19. Wienecke, E.: Anschauliche Darstellung der Hauptsätze der Planimetrie. Berlin (1902)

20. Witting, A.: Der mathematische Unterricht an den Gymnasien und Realanstalten im Königreich Sachsen. Leipzig/Berlin (1910)

21. o.V.: Der Kinematograph als Hilfsmittel im mathematischen Unterricht. Licht-Bild-Bühne 2, Nr. 9 (1912), S. 10-12

22. o.V.: Statistisches Jahrbuch der Stadt Nürnberg. Zweiter Jahrgang für 1910. Nürnberg (1911)

23. o.V.: Rundschau. Pädagog. Archiv 54, 106-115 (1912)

24. Schoemann, A.: Der deutsche Animationsfilm. Sankt Augustin (2003) 